\title{
Prognosis of Resected Neuroendocrine Metastases: A Complex Puzzle Can Only be Solved One Small Piece at a Time
}

\author{
Lana Bijelic, MD \\ Hospital Moisès Broggi Sant Joan Despí, Consorci Sanitari Integral, Barcelona, Spain
}

Many aspects of care for patients with gastrointestinal neuroendocrine tumors present challenges and uncertainties for clinicians. This is true for the entire spectrum of possible presentations spanning a wide range-from management of small, incidentally discovered duodenal or pancreatic tumors, to that of liver metastases. In this issue of Annals of Surgical Oncology, Xiang and colleagues attempt to deepen our understanding of prognosis (expressed using disease-free survival [DFS]) after curative liver resection of neuroendocrine liver metastasis by developing a novel nomogram, a first for this subgroup of patients. ${ }^{1}$ Considering the relative rarity of the disease, those researchers have assembled a robust collaborative group: six institutions provided data for the development set, consisting of 279 patients, and two institutions formed the validation set of 98 patients. The multi-institutional nature of the study and the ability to analyze prognostic factors in a large cohort is clearly one of the strengths of this study. It allowed the authors to perform a multivariable analysis and identify several prognostic variables associated with DFS.

Despite surgery being the standard of care for patients with resectable neuroendocrine liver metastases, recommended by both the National Comprehensive Cancer Network (NCCN) guidelines and the European Society for Neuroendocrine Tumors (ENETS), the identification of well-established prognostic variables associated with better outcomes after surgery has been relatively slow. ${ }^{2}$ Unlike for liver metastases from colon cancer, we lack prognostic

(C) Society of Surgical Oncology 2020

First Received: 11 May 2020;

Published Online: 20 May 2020

L. Bijelic, MD

e-mail: lana.bijelic@csi.cat scores specific for patients treated with liver resection to help inform decisions and formulate follow-up plans. In a 2019 study, Lv et al. studied prognostic factors in 108 patients with neuroendocrine liver metastases treated with various modalities, including liver-directed therapy, surgery, and systemic therapy (or combinations) and found that hepatic tumor number, treatment modality, and elevated $\mathrm{Ki}-67$ index between the metastatic and primary lesions were associated with overall survival (OS). The resulting nomogram had a $\mathrm{C}$ index of 0.63 , significantly lower than the value proposed in the study published in this current issue, which had a $\mathrm{C}$ index of 0.754 in the training cohort and 0.748 in the validation cohort. ${ }^{3}$ Furthermore, unlike the present study, Lv et al. used OS as an endpoint, which has significant drawbacks in a disease with a relatively indolent course, even in the metastatic setting. In fact, a consensus report of the National Cancer Institute Neuroendocrine Tumor Clinical Trials Planning Meeting indicated PFS as the recommended primary endpoint for clinical trials of advanced neuroendocrine tumors. ${ }^{4}$

The study in the current issue is a good step toward more clearly identifying and/or confirming clinically relevant prognostic factors specific for patients undergoing resection of neuroendocrine liver metastases. Nonetheless, it is important to consider that some findings differ, at least in part, from those found in other large cohort studies. The present manuscript highlights four clinical variables that are significantly associated with DFS on multivariable analysis: lymph node metastases, pancreatic primary, type of liver resection, and tumor grade. The identification of four variables allowed the authors to build a nomogram based on the variables' $\beta$ coefficients. This is somewhat in contrast to the findings of Sahara et al., who studied 521 patients treated at 12 international centers. ${ }^{5}$ While, on bivariate analysis, most of the variables overlap with those presented in the current study, in the report by Sahara et al., 
only lymph node status remains significant on multivariable analysis. The reasons for this difference are not immediately obvious and will need further study.

Multiple authors have found the site of the primary tumor to be prognostic. Patients with a pancreatic neuroendocrine primary fair worse than those with other gastrointestinal neuroendocrine tumors. This finding appears consistent across various potentially curative therapeutic approaches to liver metastases, from liver-directed therapy to transplantation. ${ }^{6}$ The evolution and approval process for newer systemic treatments of advanced unresectable metastatic neuroendocrine tumors (NETs) has also followed differential paths for pancreatic and gastrointestinal NETs. ${ }^{7,8}$ This was based in part on the discovery that autocrine activation of the mammalian target of rapamycin (mTOR) signaling pathway, mediated through insulin-like growth factor 1, is important for proliferation in pancreatic NETs. In addition to the mTOR pathway, several other recurrent gene mutations have been discovered in pancreatic NETs: MEN1 (which influences chromatin remodeling and gene expression), and, more recently, chromatic remodelers DAXX and ATRX, which can lead to chromosome instability. On the other hand, the genomic alterations of gastrointestinal NETs have been studied less and failed to clearly identify recurring mutations, with the exception of CDKN1B, which seems to be present in approximately $8 \%$ of small intestinal NETs. While the mutational burden of NETs is low, epigenetic changes as a potential important driver of NET progression are emerging and may open up possibilities for new therapeutic interventions. ${ }^{9}$ Overall, our understanding of the 'omic' landscape of NETs is still insufficient and it is therefore not surprising that molecular alterations have not been widely studied as clinical prognostic factors and are not included in the present study.

On the other hand, tumor grade and markers of tumor proliferative activity (such as mitotic index and Ki67) have long been identified as prognostically important in NETs and are incorporated as part of the diagnostic and staging schemas. As the current study by Xiang et al. is retrospective in nature, it relies on the 2010 WHO classification of NETs. A new edition of the WHO criteria was adopted in 2017 and brought some changes to the classification, introducing a category of G3 well-differentiated NETs. ${ }^{10}$ These tumors, morphologically and prognostically, are more likely to behave like G2 tumors even though they have a high Ki67 index ( $>20 \%)$, similar to poorly differentiated tumors (and may have therefore been classified as NECs by the 2010 WHO classification). It is unlikely that this change would greatly affect the results or performance of the proposed nomogram, but a more detailed evaluation of the impact of morphological grade and Ki67 index on
DFS could be interesting to explore in the future. However, in clinical practice, it is important to distinguish the new subgroup of well-differentiated, high-grade (G3) NETs from neuroendocrine carcinomas (NEC) because this has clear implications for therapeutic choices.

Finally, as it becomes more and more common for clinicians to consider an ever-increasing number of data points in their clinical decision-making process, including nomograms, scores, and molecular profiles, let us hope that tools that can facilitate the process will become more seamlessly integrated with electronic medical record systems that we use every day.

DISCLOSURES Lana Bijelic declares she has no conflicts of interest to declare.

\section{REFERENCES}

1. Xiang J, Zhang X, Weiss M, et al. Multi-institutional development and external validation of a nomogram predicting recurrence after curative liver resection for neuroendocrine liver metastasis. Ann Surg Oncol. 2020. https://doi.org/10.1245/s1043 4-020-08620-5.

2. Pavel M, O'Toole D, Costa F, et al. ENETS consensus guidelines update for the management of distant metastatic disease of intestinal, pancreatic, bronchial neuroendocrine neoplasms (NEN) and NEN of unknown primary site. Neuroendocrinology 2016;103:172-185.

3. Lv Y, Han X, Xu X et al. Risk factors affecting prognosis in metachronous liver metastases from WHO classification G1 and G2 gastroenteropancreatic neuroendocrine tumors after initial R0 surgical resection. BMC Cancer. 2019;19:335.

4. Kulke MH, Siu LL, Tepper JE, et al. Future directions in the treatment of neuroendocrine tumors: Consensus Report of the National Cancer Institute Neuroendocrine Tumor Clinical Trials Planning Meeting. J Clin Oncol. 2011;29:934-943.

5. Sahara K, Merath K, Tsilimigras DI, et al. Conditional diseasefree survival after curative-intent liver resection for neuroendocrine liver metastasis. J Surg Oncol. 2019;120:1087-1095.

6. Moris D, Tsilimigras DI, Ntanasis-Stathopoulos I, et al. Liver transplantation in patients with liver metastases from neuroendocrine tumors: a systematic review. Surgery 2017;162(3):525-536.

7. Yao JC, Shah MH, Ito $\mathrm{T}$ et al. Everolimus for advanced pancreatic neuroendocrine tumors. $N$ Engl $J$ Med. 2011;364(6):514-523.

8. Caplin ME, Pavel M, Ćwikła JB, et al. Lanreotide in metastatic enteropancreatic neuroendocrine tumors. $N$ Engl $J$ Med. 2014;371:224-33.

9. Di Domenico A, Wiedmer T, Marinoni I, et al. Genetic and epigenetic drivers of neuroendocrine tumors (NET). Endocr Relat Cancer. 2017;24(9):R315-R334.

10. Fang JM, Shi J. A Clinicopathologic and molecular update of pancreatic neuroendocrine neoplasms with a focus on the new world health organization classification. Arch Pathol Lab Med. 2019;143(11):1317-1326.

Publisher's Note Springer Nature remains neutral with regard to jurisdictional claims in published maps and institutional affiliations. 